\title{
Editorial to the Topical Collection: In Situ Exploration of the Ice Giants: Science and Technology
}

\author{
O.J. Mousis ${ }^{1}$ - D.H. Atkinson ${ }^{2}$
}

Accepted: 13 November 2020 / Published online: 16 December 2020

(C) Springer Nature B.V. 2020

The ice giant planets Uranus and Neptune are the outermost giant planets of our solar system, and represent a largely unexplored class of planetary objects, filling the gap in size between the larger gas giants and the smaller terrestrial worlds. The apparent size of these two planets in the sky is roughly a factor of 10 smaller than Jupiter and Saturn, making their physical properties much more difficult to characterize from Earth. The great distances to Uranus and Neptune have made exploration challenging, being limited to flybys by the Voyager 2 mission in 1986 and 1989, respectively. Therefore, much of our knowledge of atmospheric processes on these distant worlds arises from remote sensing from Earth-based observatories and space telescopes. Such remote observations cannot provide the "groundtruth" of direct, unambiguous measurements of the atmospheric composition, processes including thermal structure, cloud properties, and dynamics. Because of the physical limitations of remote observations, the deficiency of close-up measurements, and the complete lack of in situ science, the physical and atmospheric properties of Uranus and Neptune are poorly constrained and their roles in the formation, evolution, and current architecture of the solar system are not well understood.

In situ exploration of Uranus and/or Neptune fits perfectly within the ambitious scope of the ESA Voyage 2050 Programme. A Saturn entry probe proposal has already been submitted to the ESA M4 and M5 calls in 2015 and 2016, respectively, and a Saturn probe mission proposal was submitted in response to the NASA New Frontiers-4 call in 2016. Experience from these proposals shows that the development of entry probes matches well the cost envelope allocated to ESA M-class missions provided that the carrier/relay spacecraft is provided by another space agency with costs commensurate with ESA L-class and NASA

In Situ Exploration of the Ice Giants: Science and Technology

Edited by Olivier J. Mousis and David H. Atkinson

O.J. Mousis

olivier.mousis@lam.fr

1 Aix Marseille Univ, CNRS, CNES, LAM, Marseille, France

2 Jet Propulsion Laboratory, California Institute of Technology, 4800 Oak Grove Dr., Pasadena, CA, 91109, USA 
New Frontiers programs. An ideal combination would be a partnership between ESA and NASA in which ESA provides an entry probe as an important element of a more encompassing NASA New Frontiers or Flagship mission to one of the ice giants. A joint NASA-ESA Ice Giant Study Science Definition Team (SDT) was formed in 2016-2017 to investigate the best mission scenarios dedicated to the exploration of the ice giants. The conclusions of the study outline the high priority of an ice giant mission comprising both an orbiter and atmospheric probe. The mission architectures assessed by the 2017 NASA SDT showed that optimal launch windows will occur in 2029-30 for Neptune and 2030-34 for Uranus, depending on the use of Jupiter for a gravity assist. A 2018 internal ESA study, the ESA M* Ice Giant Concurrent Design Facility (CDF) study 1, shows that the technology is available in Europe to provide a probe in the framework of a joint mission with NASA. ${ }^{1}$

On 25-27 February, 2019, an international workshop co-sponsored by CNRS, CNES, and JPL was convened at the Laboratoire d'Astrophysique de Marseille, France, and dedicated to scientific and technology topics relevant to future in situ exploration of ice giant planet atmospheres, including the overall need for and requirements of ice giant system exploration in the context of future ice giant, gas giant, solar system, and exoplanetary studies. The Workshop on In Situ Exploration of the Ice Giants gathered more than eighty leading scientists and technologists who came together to discuss the current state of the art and future opportunities to make major progress in the field. The workshop comprised 65 presentations separated into sessions dedicated to the context for ice giant exploration (giant planets, exoplanets, and origins), ice giant atmospheres (composition, structure, and dynamics), ice giant interiors, needed technologies (entry systems, and radioisotope power systems), instrumentation, and mission concept options. Current views of ice giant exploration from NASA and ESA with an emphasis on potential ice giant probe studies in the framework of future NASA-ESA collaborations were also discussed.

This topical collection comprises a series of papers based on presentations and discussions from the Marseille 2019 meeting. It provides a timely and comprehensive review of the status of science, instrumentation, mission concept, and technology topics relevant to the exploration of ice giant planet atmospheres with entry probes, including scientific questions, how to achieve them, and technology challenges, and will be particularly useful as an up-to-date reference work for postgraduate students and researchers in any area touching upon the in situ exploration of giant planets and the related outstanding scientific questions.

\section{Science}

Atreya et al. discuss the deep atmosphere composition, structure, origin, and exploration, with a particular focus on critical in situ science at the ice giants. They argue that only entry probes are capable of measuring the key elements, noble gases in particular, and key isotopic ratios. These key constituents are accessible at 5-10 bars.

Aplin et al. present a discussion of electrical processes at the ice giants resulting both from convection as well as those not associated with storms. As Aplin et al. point out, ice giants are unique within the solar system due to their distance from the sun creating an environment wherein galactic cosmic rays can have a significant effect on atmospheric charging, clouds, and climate, in addition to the convection normally required for lightning.

Helled et al. discuss current knowledge of the ice giants, with a focus on internal structure and composition. The possible role of impacts on the formation and evolution of the ice giants, and the importance of improved observations of gravitational and magnetic fields, rotation rates, and deep composition and temperatures are also discussed.

\footnotetext{
${ }^{1}$ http://sci.esa.int/future-missions-department/61307-cdf-study-report-ice-giants/.
} 
Hueso \& Sánchez-Lavega explore the observed meteorology at cloud level and the deep "weather layers" of Uranus and Neptune, and show that a combination of orbital and in situ data will probably be required to answer satisfactorily outstanding questions in the behavior of their atmospheres.

Mousis et al. investigate the signatures of different delivery scenarios of volatiles to the atmospheres of ice giants. They discuss the impact of the delivery form (solids or vapors) of the volatiles, and the adopted formation scenario on the shape of the enrichment patterns in the atmospheres of Uranus and Neptune.

Mandt et al. discuss how the current composition of giant planet atmospheres provides information on how such planets formed, including a review of the current state of knowledge of noble gas abundances and isotope ratios in various solar system reservoirs and discussion of measurements needed to understand the origin of the ice giants.

Deleuil et al. review the characteristics and properties of Neptune-class exoplanets. Even though we still lack real Neptune/Uranus analogues, these exoplanets provide key observational constraints that allow the formation of the ice giants to be placed in a more general framework than the sole example of our solar system.

Cavalié et al. argue that an in situ probe like Galileo would provide us with invaluable direct ground-truth composition measurements, even if some of the condensibles will remain out of reach. They also show that thermochemistry and diffusion modeling can further help us to increase the science return from a probe.

\section{Ice Giant Mission Concepts}

Based on three decades of observations from ground- and space-based observatories, Fletcher et al. argue that the atmospheric circulation patterns derived from multi-spectral remote sensing can serve as a guide for choosing a suitable entry location for a future in situ probe mission to the ice giants.

Simon et al. review the design of in situ probes from recent ice giant mission concept studies. Including the 2013-2022 NASA Planetary Decadal Survey, there have been at least five comprehensive atmospheric probe engineering design studies performed in recent years by NASA and ESA.

Sayanagi et al. present the Small Next-generation Atmospheric Probe (SNAP), a novel small atmospheric probe designed to provide measurements of spatially variable phenomena in planetary atmospheres. With a focus on thermal structure and atmospheric dynamics, the design of a $50-\mathrm{cm}$ (diameter), $30-\mathrm{kg}$ probe is presented.

\section{From Science to Missions}

Blanc et al. show how the study of ice giant systems uniquely addresses the six key science questions about planetary systems formulated in the "Horizon 2061" foresight exercise. They identify the critical measurements and mission profiles needed and propose a notional ice giants missions road map covering four coming decades to address these questions.

\section{Instrumentation}

Aslam et al. present a design for a possible ice giant Net Flux Radiometer to measure the net atmospheric radiative flux as a function of altitude within seven spectral bands. The net flux measurement will help provide the radiative heating and cooling profiles, and therefore contribute to an improved understanding of the heat balance, dynamics, and structure of the atmosphere.

Renard et al. propose a novel instrument that provides in situ optical light-scattering measurements of aerosols in giant planet atmospheres, particularly in the ice giants. This 
instrument retrieves both the concentrations and phase functions of aerosols over 20 size classes in the $0.2-50 \mu \mathrm{m}$ diameter size range.

Vorburger et al. describe some of the shortcomings of remote sensing for exploration of planetary atmospheres, specifically focusing on the benefit of in situ mass spectrometer measurements of atmospheric chemical composition. Of particular interest is an overview of different types of mass spectrometers with a discussion of the current state-of-art.

Ferri et al., discuss the design of an Atmospheric Structure Instrument to provide the thermal structure, stability, electrical properties, acoustic properties for measuring the hydrogen ortho-para ratio, and dynamics that complement and supplement science measurements from all other probe investigations.

Simcic et al., describe NASA JPL's Quadrupole Ion Trap Mass Spectrometer (QITMS) with a particular focus on the value of QITMS for small probe measurements of noble gas abundances, noble gas isotopes, and a number of other key isotopes and disequilibrium species.

A reference model payload for an ice giant entry probe mission is presented by Atkinson, et al. with investigations designated as Tier 1 for the most essential (threshold) measurements and Tier 2 measurements for significant but less essential measurements.

\section{Technologies}

Venkatapathy et al. discuss the current readiness of a new thermal protection system for entry probes, the Heatshield for Extreme Entry Environments Technology, HEEET. For a range of possible entry velocities and flight path angles, the applicability of HEEET as a TPS system at Uranus and Neptune are discussed.

The current state of the art of European americium-241 radioisotope thermoelectric generator and radioisotope heater unit development is presented by Ambrosi, et al., including a detailed description of the technical program with a description of the design and evolution of RTGs and RHUs in the European programme.

As planning for future ice giant system exploration is now considered among the top priorities of solar system exploration by NASA and ESA, this topical collection represents a state-of-the-art survey covering the current scientific questions, technologies, instrumentation, and mission concepts related to entry probes.

Publisher's Note Springer Nature remains neutral with regard to jurisdictional claims in published maps and institutional affiliations. 